\title{
Body Worn Antenna System for Health Care related On- and Off-body Communications
}

\author{
M. Grimm and D. Manteuffel \\ Chair of Wireless Communications \\ Institute of Electrical and Information Engineering \\ Christian - Albrechts - University of Kiel \\ Kaiserstrasse 2, 24143 Kiel, Germany \\ \{Grimm, Manteuffel\}@tf.uni-kiel.de
}

\begin{abstract}
This paper presents an antenna concept which is focused on an optimized performance for on-body communications using the $5 \mathrm{GHz}$ ISM band. Primary design parameters are a strong normal polarization and a low effective antenna height. In addition, the antenna concept is capable to use the $2.7 \mathrm{GHz}$ E-UTRA band for off-body uplinks. In this context, the underlying on-body channel properties are discussed to deduce design aspects which result in optimized propagation properties. Based here on, a numerical method is used to calculate the antenna on-body radiation characteristic to determine a model of the on-body path gain. The related results show a good agreement with a numerical calculated path gain along a realistic full human body model.
\end{abstract}

Index Terms-Antenna theory, Biomedical Communication, Electromagnetic Propagation.

\section{INTRODUCTION}

One of the key aspects of upcoming health care applications are outpatient systems which aim for long-term diagnostics as well as for stressless health prevention methods [1]. Primary element of these systems is the wireless connection of wearable sensors. Such systems should be flexible enough to combine general information, like the mobility of the patient, with specialized data, e.g. body temperature, blood pressure and breathing. Especially in terms of wearable systems reliable communications between the wireless sensor nodes are required. In addition uplink properties to other wireless networks topologies, like the cellular network, are desired. Due to the vital nature of health care related applications a key element in the design process of such systems is an antenna evaluation method which allows the conception of reliable wireless applications.

The first part of the paper discusses the general properties of a line-of-sight on-body propagation channel for the ISM bands at $2 \mathrm{GHz}$ and $5 \mathrm{GHz}$. For the deduced antenna profile a corresponding design is realized in terms of a planar inverted-F antenna which is suitable to connect body worn sensors. In this context a path gain model is verified which is related to the proposed antenna concept. The second part of the paper aims at an extension of the presented antenna concept to provide uplink properties using the $2.7 \mathrm{GHz}$ LTE standard.

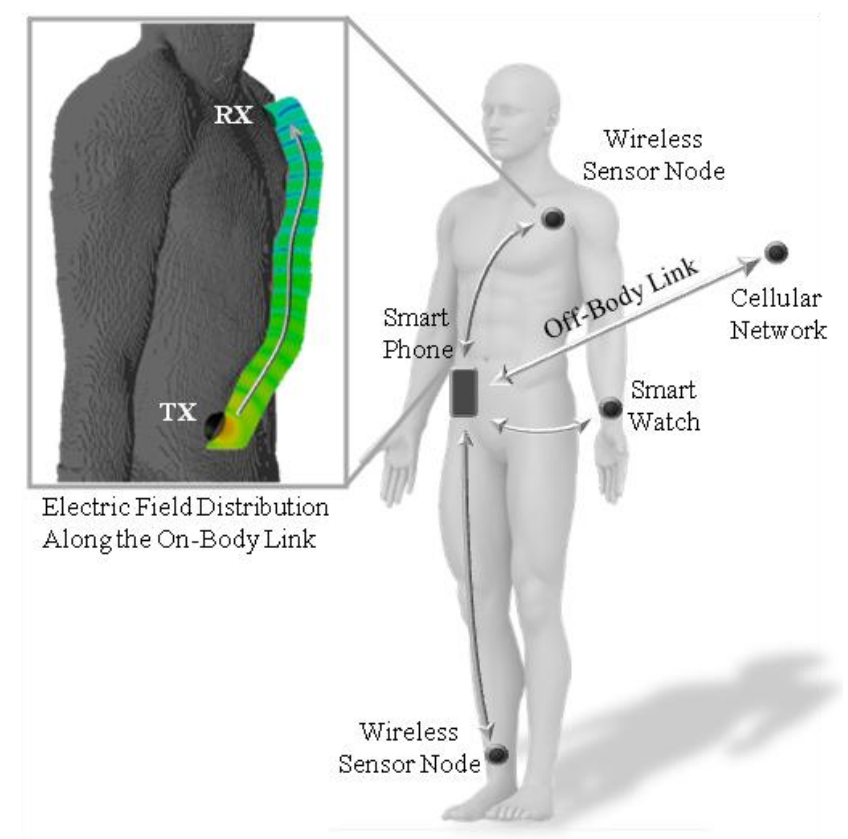

Fig. 1. Wireless connection of worn medical devices by different onbody links and off-body link for data upload into the cellular network; numerical calculations are based on [2] and [3].

\section{ON-BODY CHARACTERIZATION OF BODY WORN ANTENNA SYSTEMS}

A demanding problem in the design process of body worn antennas is the connection of an explicit antenna geometry, inclusive the related electrical excitation, to the corresponding on-body far field. In general, the close proximity of the antenna to the human body prohibits the usage of established theoretical approaches which are based on free space propagation conditions. In order to treat this problem, an approach has been developed to describe the on-body radiation characteristic of antennas consisting of a perfect conductor which are positioned in close proximity to the human body [4]. 


\section{A. On-Body Channel Characteristic in the $2.45 \mathrm{GHz}$ and $5.4 \mathrm{GHz}$ ISM Band}

The fundamental basis of the developed theory are terrestrial far field models which were developed in the last century. Primary assumption of these models is an infinite flat ground where the antenna is positioned at a low effective antenna height above the surface, i.e. the spacing is small in terms of the wavelength. A possible solution of this problem has been developed by Bannister. The corresponding theory enables a description of the electromagnetic field of basic dipole antenna types ranging from the quasi-static range to far field distances. In the scope of body centric communications it has been shown that his approach can be adapted to deduce general on-body channel characteristics [5]. The related mathematical formulation allows a direct insight into the related radiating mechanisms and enables a discussion of the field values as a function of constitutive parameters, e.g. polarization, frequency and effective height. Especially, by categorizing the total radiated electromagnetic field into two components, namely the space wave and the Norton surface wave, a direct deduction of the wave form has been enabled. In this context, the dielectric parameters of the ground have been set in conjunction with the internet data base [6] for the following discussions to muscle tissue, compare Table 1.

TABLE I

ASSUMED DIELECTRIC PROPERTIES OF THE GROUND

\begin{tabular}{ccccc}
\hline \hline Symbol & Quantity & Unit & \multicolumn{2}{c}{ Value } \\
\hline$f$ & frequency & $\mathrm{GHz}$ & 2.45 & 5.4 \\
\hline$\varepsilon_{\mathrm{r}}$ & relative permittivity & 1 & 52.7 & 49.0 \\
\hline$\sigma$ & conductivity & $\mathrm{Sm}^{-1}$ & 1.74 & 4.49 \\
\hline \hline
\end{tabular}

Related to the focused on-body scenario Fig. 2 shows the ratio of the space wave to the Norton surface wave. In this context, a value below one implies the excitation of a strong Norton surface wave, i.e. the radiated electromagnetic wave tends to be guided by the curvature of the body surface. On the other hand side a strong space wave tends to be reflected away from the body. Moreover, the results indicate that a tangential antenna polarization is not capable to excite a dominant surface wave at all, and it is shown that the magnitude of the Norton surface wave is higher for lower effective antenna heights.

\section{B. On-Body Antenna Design}

In conjunction with the deduced results a planar inverted-F antenna design has been chosen which combines a strong normal polarization with a low effective antenna height. In addition, the relative large metallic ground plane aims at shielding the antenna feed from the human body. By this, local variations of the human tissue distribution have less significant influence on the antenna return loss. Other geometry parameters of the antenna, see Fig. 3, are optimized in the way that the antenna return loss is below $-10 \mathrm{~dB}$ within the referred WLAN frequency band $(5150 \mathrm{MHz}-5350 \mathrm{MHz})$.
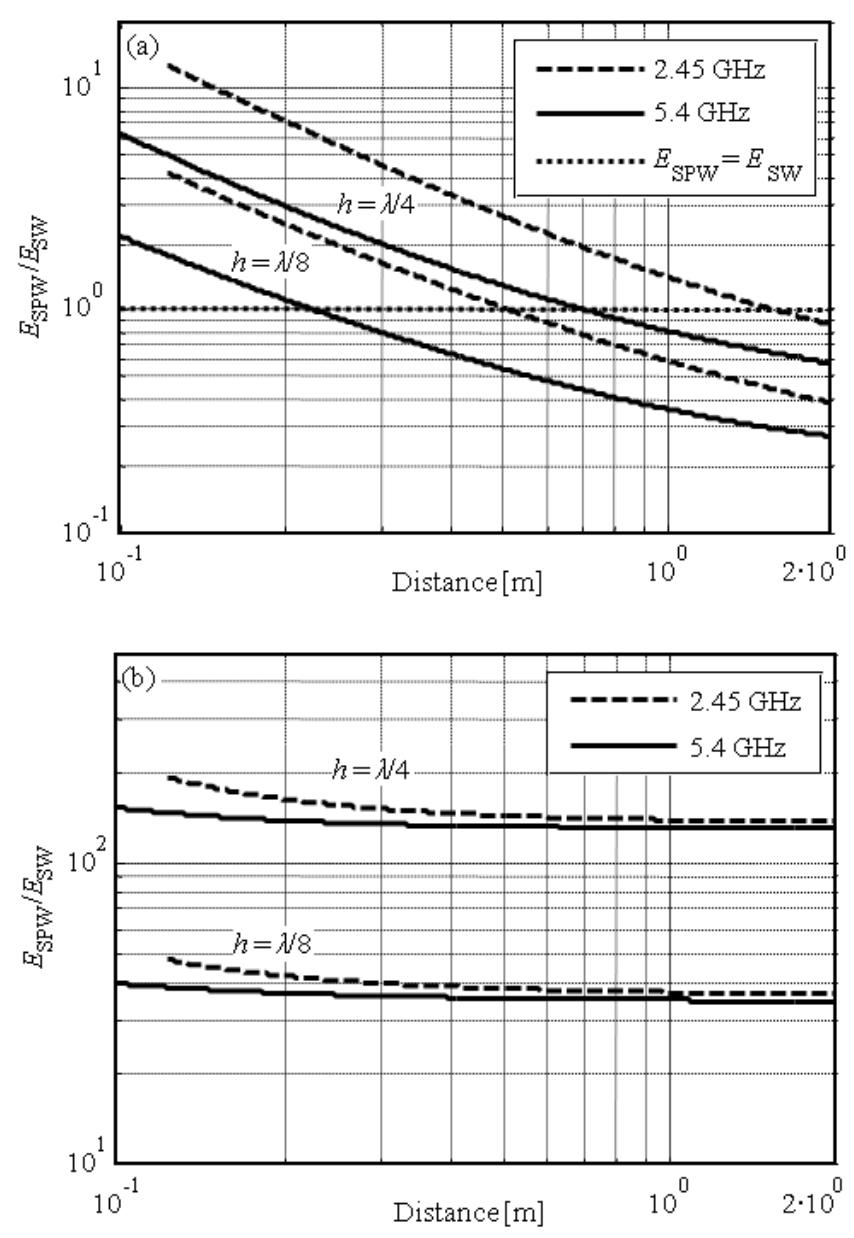

Fig. 2. Ratio of the space wave to the Norton surface wave above a flat and homogeneous muscle tissue body; (a) normal polarization; (b) tangential polarization

As shown in Fig. 3 a homogeneous flat muscle tissue ground has been considered to take into account the influence of the human body in terms of the antenna design. To prevent a leaking of the excited antenna current distribution on the metal components into the human body a separation distance of $1 \mathrm{~mm}$ is applied. In a real design this fact would be realized by a plastic housing of the application. (a)

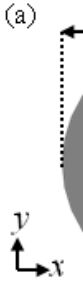

34.0

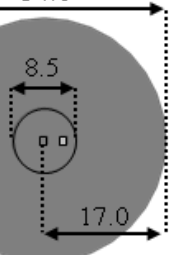

(b)

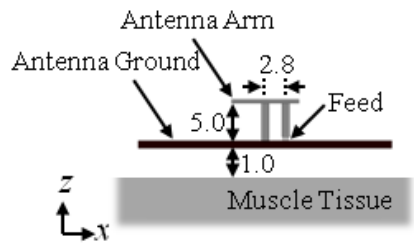

Fig. 3. Planar inverted-F antenna designed for on-body communication links at $f=5.4 \mathrm{GHz}$; (a) Top view; (b) Side view inclusive of body phantom

\section{Estimation of the effective antenna height}

Despite the strong normal antenna polarization the on-body far field cannot be directly estimated by the antenna geometry. Nevertheless, a method has been used to describe the antenna 
on-body far field by a numerical near field calculation [7]. The underlying approach segments the current density distribution of the excited antenna structure into a finite number of $N$ space cells at the position $\mathbf{r}_{0, \mathrm{i}}$ with $i \in[1, N]$. Here, each cell represents an electric small dipole which is weighted by the factor $\mathbf{w}_{\mathrm{i}}$ and is related to the current density value of the cell. The corresponding Cartesian coordinate system has been chosen such that the $z$-direction unity vector is normal orientated to the body surface. For each cell the corresponding on-body far field is calculated by the Bannister equations [5]. The total radiated on-body antenna far field is then constructed by the weighted superposition of all dipole elements.

The description of the antenna current by this method enables even a relative simple estimation of the effective antenna height $h$. For this purpose the $z$-component of each cell is multiplied by the corresponding weighting factor and summed up. The effective height results then by the normalization to the total sum of the weight factors, i.e. by the following equation:

$$
h=\frac{\sum_{i \in[1, N]} w_{z, i} r_{0, z, i}}{\sum_{i \in[1, N]} w_{z, i}} .
$$

\section{On-Body Path Gain}

With the method described above the Bannister equations allow a calculation of the electric and magnetic on-body far field. Considering Poynting's theorem this enables even a discussion of the power flux density $\mathbf{S}$ along the body surface. In order to calculate the path gain of a propagation scenario which consist of two of the developed on-body antennas the power flow flux density at the effective antenna height has to be weighted by the effective antenna area.

An estimate of the effective antenna area $A_{\text {eff }}$ is derived from a numerical FDTD calculation where the receive antenna (RX) is positioned in the far field of the transmit antenna (TX). In this case the spacing has been set to $0.15 \mathrm{~m}$, i.e. a distance of nearly three free space wave lengths has been chosen. In such a case it has been shown that the ratio of the received output power to the power flux density of the transmitting antenna is even for a farther distance nearly constant [5]. The corresponding value has been calculated by (2) as follows:

$$
A_{\text {eff }}=\frac{\left|P_{\mathrm{RX}}(d=0.15 \mathrm{~m})\right|}{\left|S_{\text {Model }}(d=0.15 \mathrm{~m})\right|} \simeq 42 \cdot 10^{-3} \mathrm{~m}^{2}
$$

The validity of this approach is shown in Fig. 4 where the modeled receive power is shown in comparison to three independent numerical calculated setups with spacing $0.5 \mathrm{~m}$, $0.6 \mathrm{~m}$ and $0.7 \mathrm{~m}$ to the transmitting antenna.

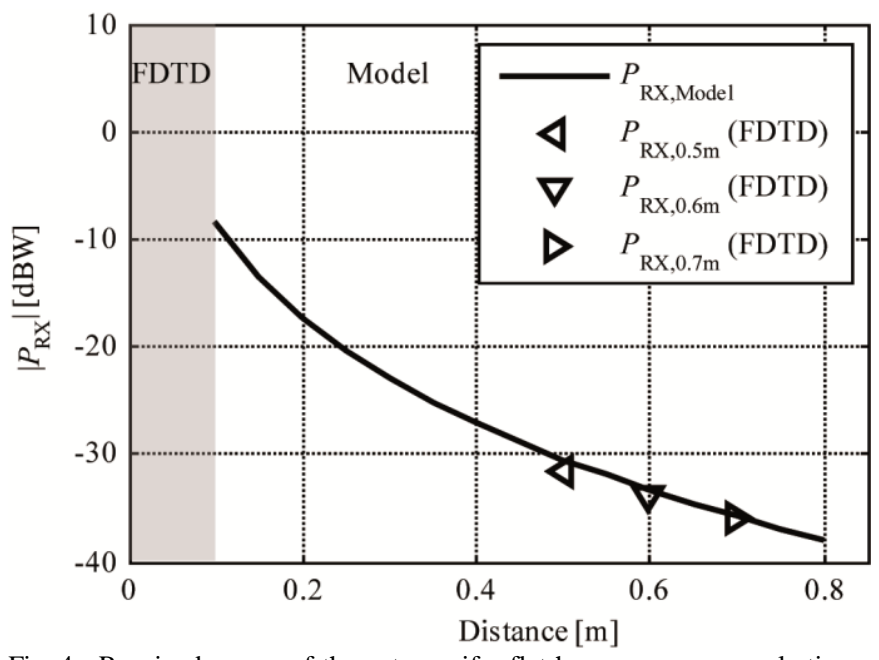

Fig. 4. Received power of the antenna if a flat homogeneous muscle tissue ground is assumed. Solid line: Model; Discrete values: FDTD

Using (2) the corresponding path gain $P G$ for arbitrary far field distances can be calculated by:

$$
P G=\frac{P_{\mathrm{RX}}}{P_{\mathrm{TX}}}=\frac{\left|S_{\text {Model }}\right| A_{\mathrm{eff}}}{P_{\mathrm{TX}}}
$$

\section{E. Validation by a Realistic Propagation Scenario}

Due to the origin of the Bannister equations the presented results are restricted to ideal propagation scenarios in principle, i.e. the modeled body has to be infinite, flat and homogeneous. Those three criteria are generally not given in the context of general body centric communication applications. Using the realistic human body phantom 'Duke' [3] a corresponding on-body scenario has been numerically calculated to verify the results. The evaluated propagation path leads from the right side of the hip along the leg to the ankle. At each distance point the antenna structure has been adjusted such that the antenna ground is aligned parallel to the body surface.

A comparison of the results shown in Fig. 5 reveals that the modified ground wave theory used is capable to model the average mean value of the far field. As a matter of fact if the direct line-of-sight between transmitting and receiving antenna is blocked by local obstacles, e.g. by the curvature of the knee, the presented approach can be used to model the average mean value of the path gain. Even at the largest distance, where the antenna has to be rotated by about $45^{\circ}$ to ensure an adequate antenna alignment to the body surface, the results look acceptable. This behavior can be reduced to the nature of the Norton surface wave which is connected to the boundary between body and free space and therefore guided by the body surface. 


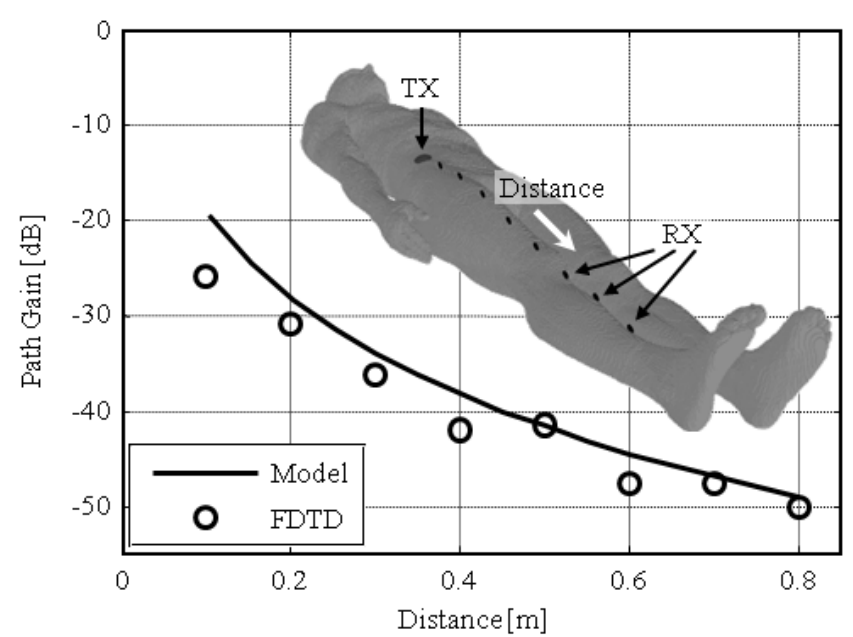

Fig. 5. Path gain along an on body path which leads from the hip along the right leg to the ankle. Solid line: Model values; Circles: Position of the receiving antenna for the FDTD based validation values.

\section{OFF-BODY CHARACTERIZATION OF BODY WORN ANTENNA SYSTEMS}

The proposed on-body antenna is optimized in the way that a strong Norton surface wave is excited. This may results in poor propagation properties for links which are directed away from the human body, the so called off-body links. As seen in Fig. 6 the developed on-body antenna layout has been modified by an additional antenna arm. To reduce the interaction of the two antenna feeds the ground below the offbody antenna arm has been slotted by seven cut-ins of $1 \mathrm{~mm}$ width.

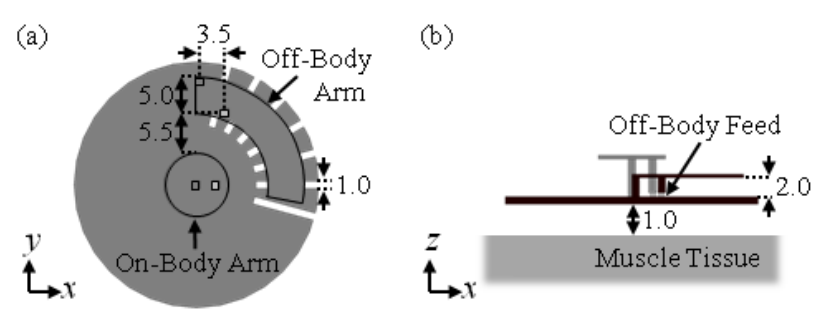

Fig. 6. Extension of the Planar inverted-F antenna design for off-body communication links at $f=2.7 \mathrm{GHz}$; (a) Top view; (b) Side view inclusive body phantom.

The design of the arm aims for a stronger space wave excitation at the E-UTRA band $7(2500 \mathrm{MHz}-2690 \mathrm{MHz})$ by a stronger tangential antenna polarization. The realized return loss within the frequency band is below $-10 \mathrm{~dB}$ while the return loss of the on-body feed is nearly unaffected by the chosen antenna extension, see Fig. 7. Therefore, a medical onbody device using this enhanced antenna design may act as a relay to connect several wireless on-body sensor nodes to an external off-body network, e.g. with the presented smart phone operating in the $2.7 \mathrm{GHz}$ E-UTRA band.

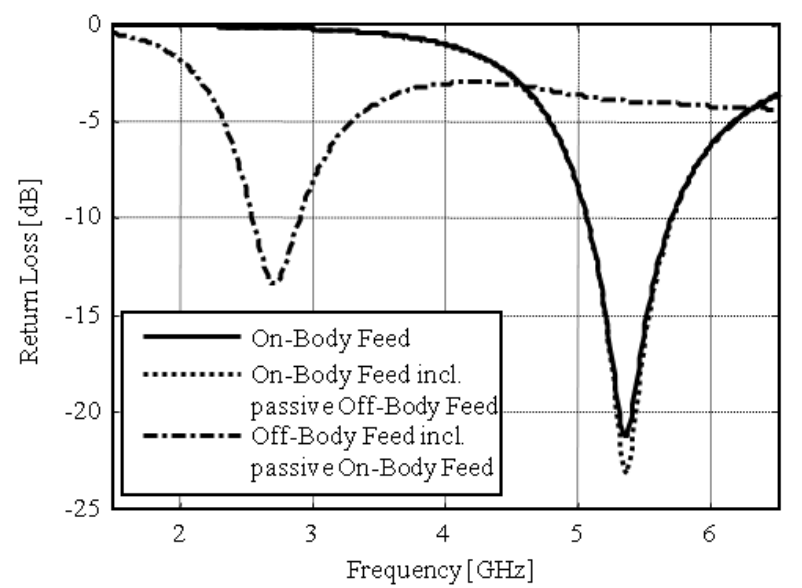

Fig. 7. Return loss of the on-body antenna with and without the off-body extension.

\section{CONCLUSION}

The presented antenna design shows good qualities for onbody as well as for off-body communications. In addition, the underlying design rules are flexible enough to be adapted to other applications, e.g. other frequency bands, by minor layout adjustments. Even in terms of realistic scenarios the path gain model technique enables sufficient estimations of the average reaction of the propagation channel. Therefore, the influence of certain antenna design parameter can be evaluated in the context of requested application properties.

\section{REFERENCES}

[1] P. S. Hall, and Y. Hao, "Antennas and Propagation for Body-Centric Wireless Communications," Artech House, 2006.

[2] EMPIRE XCcel website, [Online], Available: http://www.empire.de

[3] IT'IS Foundation website, [Online]. Whole-Body Human Models, Enhanced Anatomical Models, Available: http://www.itis.ethz.ch/

[4] M. Grimm, D. Manteuffel, "Far Field Modeling of Body Worn Antennas by the Superposition of Equivalent Electric Sources," Antennas and Propagation (EuCAP), Eighth Conference on, The Hague, 2014

[5] M. Grimm, D. Manteuffel, "Norton Surface Waves in the Scope of Body Area Networks," Antennas and Propagation, IEEE Transactions on , vol.62, no.5, pp.2616,2623, May 2014

[6] An Internet Resource for the Calculation of the Dielectric Properties of Body Tissues, The Institute for Applied Physics, Italian National Research Council website. [Online]. Available: http://niremf.ifac.cnr

[7] M. Grimm, D. Manteuffel, "Evaluation of the Norton equations for the development of body-centric propagation models," Antennas and Propagation (EuCAP), Sixth Conference on, Prague, 2012 